\title{
The design of the copper electrolyte temperature and cell voltage monitoring system
}

\author{
Cheng Liu, Lichun Shi , Tianyu Wang \\ College of Electrical Engineering, Beihua University,Jilin 132021 China \\ slcacy@163.com
}

\begin{abstract}
Keywords: MCIMX287CVM4B; Wireless transmission; Data Acquisition; CAN Bus; Electrolytic Copper
\end{abstract}

\begin{abstract}
This paper introduces a temperature of copper electrolyzer and voltage acquisition system which is divided into three layers, lower computer collects cell temperature and voltage using MCIMX287CVM4B, then sent to the gateway basing on the MCIMX287CVM4B through wireless network, in the end the gateway transfers data to the host computer via CAN bus. The paper also introduces the method to solve the general design scheme of the system and key technology. The system has high accuracy, good stability, a higher value and promotional value.
\end{abstract}

\section{Introduction}

In copper electrolysis production, electrolyte temperature and voltage on the cell has close relationship with production quality, technology personnel need to adjust process parameter according to the temperature of electrolyte and voltage of electrolyzer. The traditional production, in order to grasp the temperature of electrolyte and voltage of electrolyzes, production personnel to the scene with the instrument need timing manual measurement the temperature and voltage, Electrolytic cell number as many as hundreds of thousands of workshop, and the groove surface of high temperature, acidic liquid corrosion resistance, poor working conditions, Each electrolytic cell measurements again is a time-consuming and laborious work, and this measurement is difficult to reflect the parameters of electrolyzes cell in time.

The voltage at both ends of the cell referred to as 'cell voltage', cell voltage is another parameter that relevant to electrolytical copper quality, change of cell voltage reflects the electrolytic conditions of electrolytic cell. According to the change of cell voltage, can determine whether the existence of the cathode plate and the anode plate short circuit condition, whether the presence of copper electrode poor contact conditions. Electrolytic copper production process, in order to discover short plate, production personnel need regular drag on the groove face Gauss meter, which is also a difficult and tedious work, spend a lot of labor costs.

The voltage and temperature acquisition system of electrolytic cell , is proposed in this paper can be real-time and efficient acquisition of production process the data needed, greatly reduce the labor intensity of workers, improves the production efficiency.

This system consists of three parts: data acquisition node, communication gateway, host computer. The system structure is shown in figure 1. By the 64 detection device (data acquisition), the 8 gateway, a CAN bus communication card and a host component. Each detector means for measuring the parameters of eight electrolytic cell , each gateway is responsible for eight detection device, the host and the gateway through the CAN bus connection. 


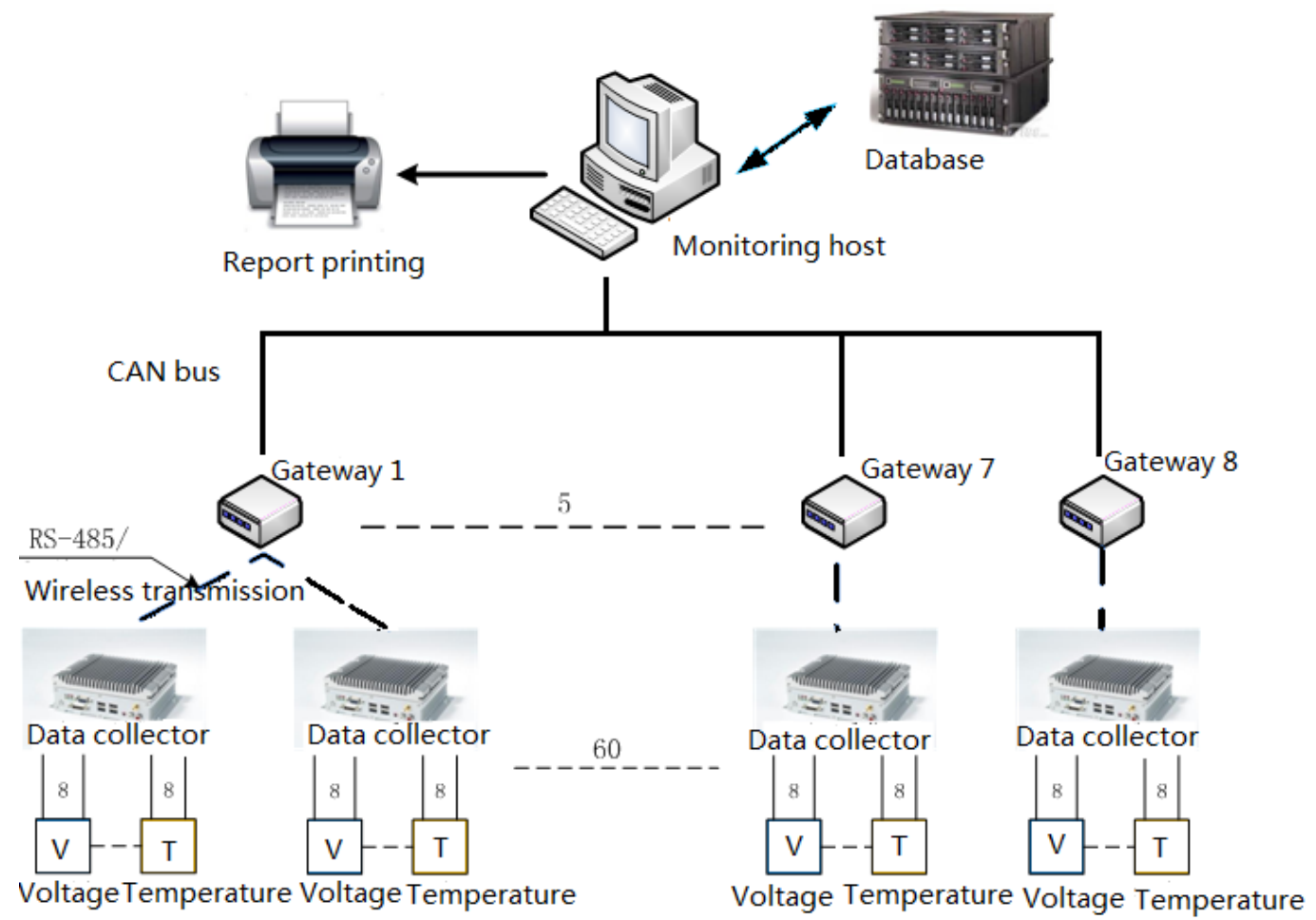

Fig. 1 Electrolytic cell temperature and voltage monitoring system structure

\section{System Overview}

The system uses Philips company's ARM9TDMI-S ARM microprocessor core MCIMX287CVM4B. The processor has a smaller 289 pins BGA encapsulation, extremely low power consumption and small volume. It has $128 \mathrm{~KB}$ static RAM, $128 \mathrm{~KB}$ on-chip programmable ROM, 8 road 12 bit ADC, multiple internal interrupt, four 32-bit timers, 8-way output PWM unit such as on-chip resources, especially suitable for industrial control and medical system and so on. The design uses it, can meet the requirements of miniaturization, low power consumption, low cost, power data acquisition nodes and gateway were below $1 \mathrm{~W}$.

Data acquisition node in a MCIMX287CVM4B as the core, can collect the 8 electrolyzer voltage and temperature signal, each cell temperature is divided into front and rear ends. The voltage signal after the transformation, the conversion from MCIMX287CVM4B chip AD converter for digital signal; a temperature sensor DS18B20, the digital signal can be directly obtained temperature. Data acquisition is completed, by the MCIMX287CVM4B through the wireless transceiver module STR -30 is sent to the gateway.

Communication gateway to the same MCIMX287CVM4B as the core, data acquisition node is transmitted by the wireless communication module STR-30 receive data. Because the cell may be numerous, so each gateway with only eight data acquisition node communication, this can avoid too far distance caused by the communication is not reliable, but also can shorten the cycle time of a single data acquisition device.

PC using Configuration software graphical programming language to complete the display interface design, can show the changes of temperature and voltage of each electrolytic cell in real time, and can set the alarm, check the history curve and history alarm, data statistics and print.

\section{Data acquisition node}

Each data acquisition node can collect 8 electrolytic cell data, including the cell voltage and front and rear end temperature, structure diagram as shown in Figure 2. 


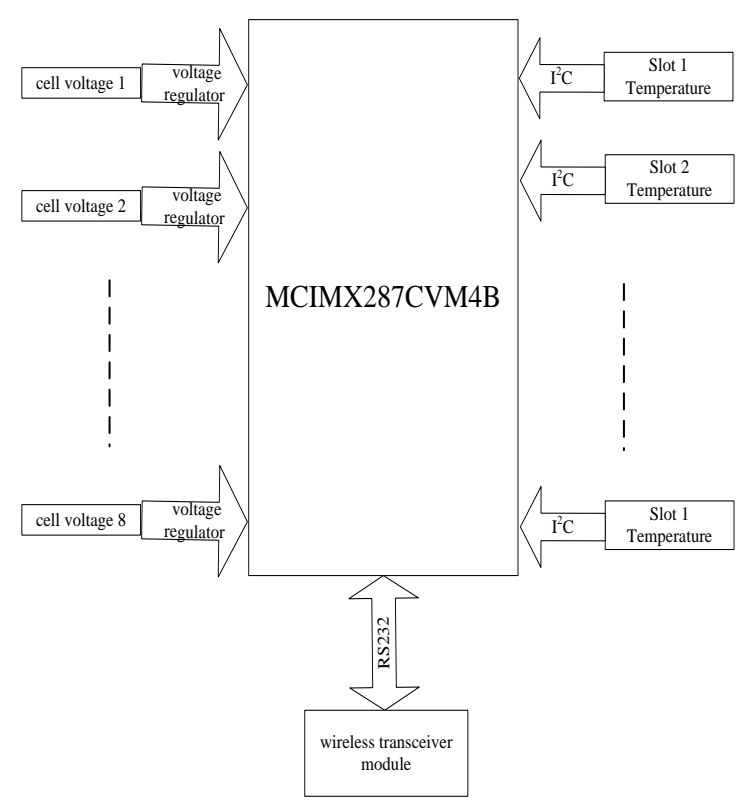

Fig. 2 Data acquisition node structure

Cell voltage is very low, generally only about $0.3 \mathrm{~V}$, this signal is susceptible to noise, and therefore, filtering and amplification is required in $\mathrm{AD}$ conversion before. Temperature sensor DS18B20 and MCIMX287CVM4B are connected by IIC bus, 16 bit digital measure the temperature with volume is the direct use of MCIMX287CVM4B.

In with the gateway communication mode, select STR-30 wireless transceiver module, select the wireless transceiver module STR-30. The wireless transceiver module has the advantages of convenient use, long communication distance, the theory of communication distance can be up to 800 $\mathrm{m}$, in actual use, in order to ensure the reliability of communication, each gateway and data acquisition node communication distance of not more than $200 \mathrm{~m}$, this communication mode greatly reduces the wiring work, while in use, difference and cable serial port is not, as shown in Figure 3.

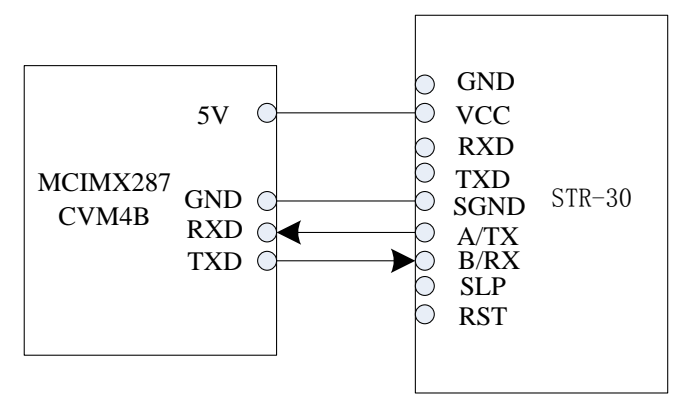

Fig. 3 STR - 30 and MCIMX287CVM4B connection diagram

STR - 30 use from $2.7 \mathrm{~V}$ to $5.5 \mathrm{~V}$ DC power supply, the power supply can be directly used MCIMX287CVM4B. Emission current acuities were $40 \mathrm{~mA}$ at work, receives current $30 \mathrm{~mA}$ or less, sleep current 20 uA or less. Data acquisition node software flow as shown in Figure 4.

\section{Design of the detection unit cell voltage}

Cell voltage detection unit function diagram as shown in Figure 5, including: signal conditioning and overvoltage protection, digital isolation sampling, data display, control and communication module.

The detection unit using ARM9 processor core technology, with high speed and rich peripherals, the design is simple, the debugging more convenient. The detection unit design of RS-485 industrial field bus, using isolation technology, which can provide high-speed access and networking function .

A 12 bit high-speed SPI acquisition can ensure the accuracy of sampling, provides valuable data for the digital filter. The use of photoelectric isolation technology, electrical contact isolation of 
analog and digital components, improves the reliability and safety of the detection unit. LED digital circuit shows 8 voltage parameters, alarm or control output can be realized through isolated digital IO.

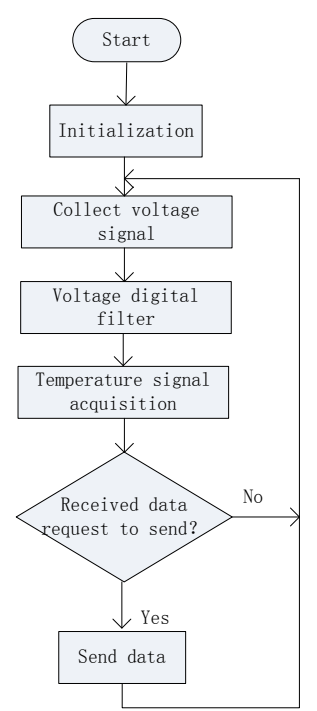

Fig. 4 Data acquisition flowchart

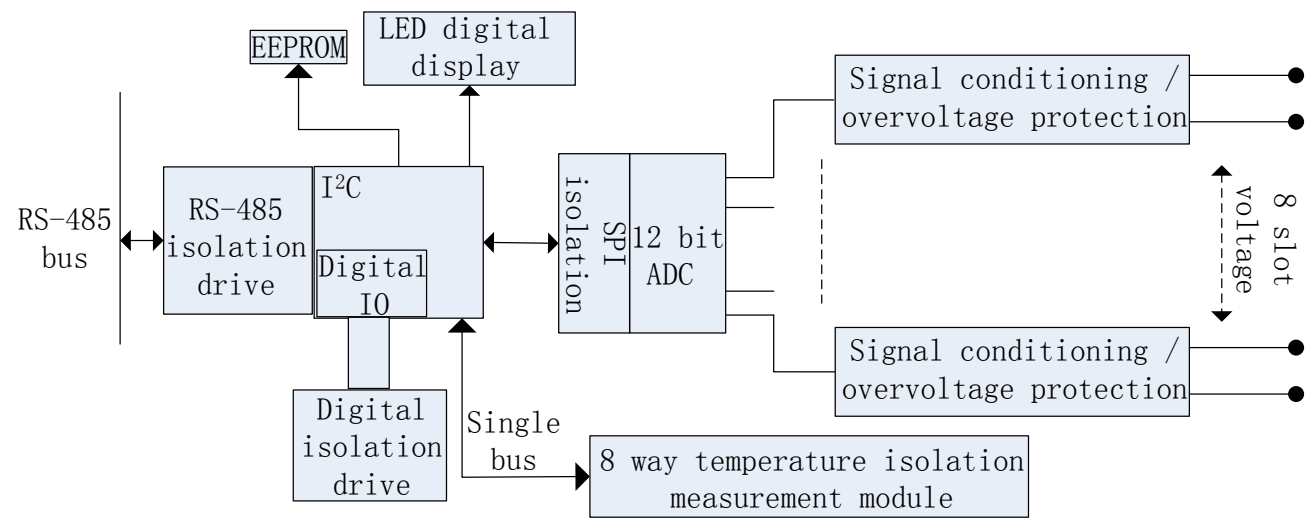

Fig. 5 Cell voltage detection unit function diagram

\section{Design of Electrolytic cell temperature detection module}

Electrolytic cell temperature detection module principle diagram as shown in Figure 6, each data collector is connected with 8 DS18B20 digital temperature sensor to realize the electrolytic cell temperature signal acquisition.'single bus' design can reduce wiring complexity, solved the analog transmission under strong magnetic field are susceptible to interference.

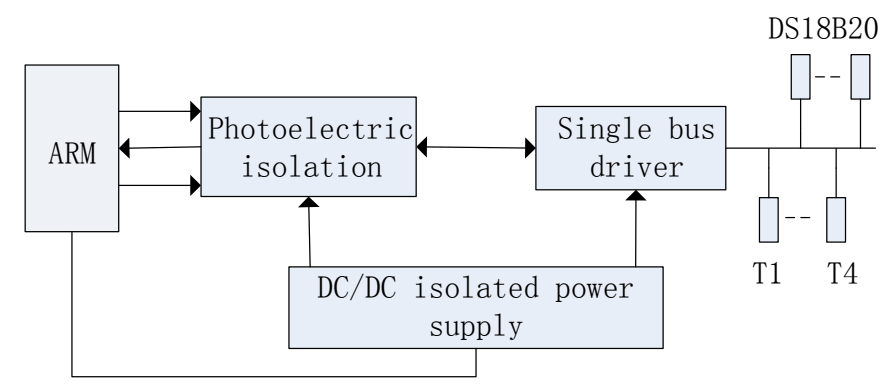

Fig. 6 electrolyzer tank temperature detection module schematics

\section{PC screen real-time monitoring software}

Real time monitoring of PC software using Configuration to develop, with digital, curve display, historical data storage and query statements, functional data analysis, alarm etc. 


\section{The gateway}

A gateway is a data acquisition device and PC data transfer station. Between the gateway and the data acquisition node by wireless way communication between PC and using the CAN bus communication. CAN bus of the effective communication distance, strong anti-interference ability, and is very suitable for copper electrolysis workshop working environment. Each gateway to the cycle of 8 data detecting nodes request data, receive data, the data is temporarily stored in RAM, the request data to the gateway to wait for the host computer; received the PC data request, through the CAN bus to the PC to send 8 detection node voltage and temperature signal.

\section{Conclusion}

This data acquisition system provides a kind of the electrolytic cell parameters monitoring system with wireless transmission device, the electrolytic cell parameters on-line detection, control, achieving high stability and high reliability operation, The application of this system not only greatly reduce the work intensity of the production personnel, and also provides a timely process engineering analysis of electrolytic product quality and reliable field parameters, analysis of improvement of product quality, reduce electricity consumption and manpower cost. This monitoring system technology is advanced, the function expansion is convenient, simple maintenance, has a certain application promotion value.

\section{Acknowledgements}

This work was financially supported by the National Natural Science Foundation of China (61179012), Educational Commission of Jilin Province of China (2014167) and Program for Innovative Research Team of Jilin Province of China (20150519023JH).

\section{References}

[1] Wenlin Zhang. Introduction to copper electrolytic refining of electrode short circuit[J] Nonferrous metallurgy and energy saving.2006.26.

[2] Jing Yang. CAN bus interface circuit[J]Office automation.2010(02).

[3] Liang Ma.Research and application of temperature control system based on ARM[D]Taiyuan university of science and technology.2012.

[4] Haoping He,Peng Lu. Copper electrolysis production management information system architecture analysis and application.[J] Non-ferrous metallurgical design and research.2013(03).

[5] Lili Cui Zetao Li. A kind of wireless communication based on CAN bus node research[J]The modern machinery.2009(03) .

[6] Micropower wireless data transmission module USES the manual. Sunbow Electronic Technology Co., Ltd.

[7] Wenjun Luo,Zhenhua Zhao. Temperature Monitoring System Based on CAN Bus[J]. Industrial control computer.2011(05). 